\title{
Injectable Peptide Decorated Functional Nanofibrous Hollow Microspheres to Direct Stem Cell Differentiation and Tissue Regeneration
}

\author{
Zhanpeng Zhang, Melanie J. Gupte, Xiaobing Jin, and Peter X. Ma*
}

Injectable microspheres are attractive stem cell carriers for minimally invasive procedures. For tissue regeneration, the microspheres need to present the critical cues to properly direct stem cell differentiation. In natural extracellular matrix (ECM), growth factors (GFs) and collagen nanofibers provide critical chemical and physical cues. However, there have been no reported technologies that integrate synthetic nanofibers and GFs into injectable microspheres. In this study, functional nanofibrous hollow microspheres (FNF-HMS), which can covalently bind GF-mimicking peptides, are synthesized. Two different GF-mimicking peptides, Transforming Growth Factor- $\beta 1$ mimicking peptide Cytomodulin (CM) and Bone Morphogenetic Protein-2 mimicking peptide P24, are separately conjugated onto the FNF-HMS to induce distinct differentiation pathways of rabbit bone marrow-derived mesenchymal stem cells (BMSCs). While no existing biomaterials are reported to successfully deliver CM to induce chondrogenesis, the developed FNF-HMS are shown to effectively present CM to BMSCs and successfully induced their chondrogenesis for cartilage formation in both in vitro and in vivo studies. In addition, P24 is conjugated onto the newly developed FNF-HMS and is capable of retaining its bioactivity and inducing ectopic bone formation in nude mice. These results demonstrate that the novel FNF-HMS can effectively deliver GF-mimicking peptides to modulate stem cell fate and tissue regeneration.

\section{Introduction}

Stem cells are an excellent cell source for engineering human tissue substitutes because of their fast proliferation rate and

\author{
Z. Zhang, M. J. Gupte, Prof. P. X. Ma \\ Department of Biomedical Engineering \\ University of Michigan \\ Ann Arbor, MI 48109-1078, USA \\ E-mail:mapx@umich.edu \\ Dr. X. Jin, Prof. P. X. Ma \\ Department of Biologic and Materials Sciences \\ University of Michigan \\ Ann Arbor, MI 48109-1078, USA \\ Prof. P. X. Ma \\ Macromolecular Science and Engineering Center \\ University of Michigan \\ Ann Arbor, MI 48109-1078, USA \\ Prof. P. X. Ma \\ Department of Materials Science and Engineering \\ University of Michigan \\ Ann Arbor, MI 48109-1078, USA
}

DOI: $10.1002 / \mathrm{adfm} .201402618$ wide differentiation potential. ${ }^{[1]}$ To harvest the regenerating potential of the stem cells, advanced materials should be designed to present the key signals of the natural cellular microenvironment to the stem cells and direct their differentiation. ${ }^{[2]}$ Growth factors (GFs) are critical chemical signals in the extracellular matrix (ECM), which are activated during tissue development or repair to regulate cell migration, adhesion, proliferation, and differentiation. ${ }^{[3]}$ To effectively regulate stem cells, GFs should reach the cells without denaturing and remain there for a sufficient time period. ${ }^{[4]}$ Therefore, controlled spatiotemporal presentation of GFs is desired in regulating stem cells for tissue regeneration. ${ }^{[5]}$

Tethering GFs on biomaterials is a promising strategy to deliver sustained and localized GF signals to cells. ${ }^{[6]}$ Similar to ECM where GFs such as Transforming Growth Factor- $\beta 1$ (TGF- $\beta 1$ ) and Bone Morphogenetic Protein-2 (BMP-2) are bound and localized, ${ }^{[7]}$ synthetic scaffolds may also be tethered with GFs to deliver sustained signals to the local cells. The immobilization of GFs could slow down their degradation/internalization rate. ${ }^{[8]}$ Compared to the delivery of soluble GFs through adsorption or encapsulation into biodegradable polymers, ${ }^{[9]}$ covalent immobilization may likely reduce burst release that might cause toxicity to cells, ${ }^{[10,11]}$ and prevent GF diffusion to unintended surrounding tissues, which might cause complications. ${ }^{[12]}$ However, the conformation of GFs could be compromised during the covalent binding reactions, resulting in reduced bioactivity. ${ }^{[13]}$

Alternatively, covalently tethering GF-mimicking short peptides instead of the entire GF onto biomaterials is likely advantageous. ${ }^{[14]}$ These GF-mimicking peptides, derived from GFs or discovered by screening techniques, are able to recognize the corresponding GF receptors, trigger associated signal transduction and lead to similar cell responses to those by the native GFs. ${ }^{[15-19]}$ Unlike GFs which require a tertiary structure for activity, these GF-mimicking peptides typically have a substantially smaller molecular size and a linear structure, so that bioactivity can be well retained after chemical reactions. ${ }^{[16,17,19,20]}$ However, the use of these GF mimics for stem cell regulation and tissue regeneration remains largely unexplored ${ }^{[16,20]}$ because of the lack of appropriate ways to present them on 
3D scaffolds. For example, Cytomodulins (CM) are a family of TGF- $\beta 1$ mimicking peptides. Like TGF- $\beta 1$, CM can enhance the expression of collagen I and improve wound healing effect of fibroblasts. ${ }^{[18,19]}$ However, unlike TGF- $\beta 1$, the soluble form of CM peptides cannot effectively induce chondrogenic differentiation of stem cells. ${ }^{[2]}$ We hypothesized that ECM-mimicking scaffolds would enhance the bioactivity of bound GF-mimicking peptides in directing stem cell differentiation. This study was designed to develop an advanced injectable ECM-mimicking scaffold and evaluate its efficacy in enhancing bioactivity of the bound GF-mimicking peptides.

To mimic the physical structure of collagen in extracellular matrix (ECM), synthetic nanofibers have been developed, which are found to improve stem cell attachment, proliferation, and differentiation along various lineages when appropriate biochemical cues such as GFs are present. ${ }^{[22,23]}$ Therefore, we specifically hypothesized that nanofibers are excellent substrates for GF-mimicking peptides, and the conjugation may synergize the geometrical cues and the chemical cues to enhance the efficacy of GF-mimicking peptides. Current approaches to fabricating functional nanofibers include the self-assembly of peptide amphiphiles into nanofibers, ${ }^{[24]}$ electrospinning of nanofibers from functional polymers, ${ }^{[25]}$ and surface modification of nanofibers after electrospinning. ${ }^{[25]}$ However, these approaches toward tissue engineering applications have been primarily limited to in vitro studies due to the difficulty in generating controllable 3D pore structures. ${ }^{[25]}$ While novel phase-separation techniques have been shown capable of generating nanofibrous materials with well-controlled 3D pore structure, ${ }^{[26]}$ these porous 3D scaffolds are not injectable and cannot be used for minimally invasive procedures to regenerate small and irregularly shaped tissue defects. Our laboratory recently developed injectable polymeric nanofibrous hollow microspheres (NF-HMS), ${ }^{[27]}$ which, however, lack functional groups for conjugating biomolecules.

In this work, we successfully synthesized a novel functionalized graft copolymer that can self-assemble into functional nanofibrous hollow microspheres (FNF-HMS) and conjugate biomolecules such as peptides. The NF structure of the FNFHMS presents the geometrical features of collagen in natural ECM, which may enhance the efficacy of GF signals in stem cell differentiation. ${ }^{[28]}$ Two different GF mimics, a TGF- $\beta 1$ mimicking peptide CM10 and a BMP-2 mimicking peptide P24, were separately conjugated onto the novel FNF-HMS, and were evaluated for enhancing cartilage and bone regeneration, respectively.

\section{Results}

\subsection{Functionalizing PLLA-based Copolymers with Acrylic Groups}

Poly(L-lactic acid) (PLLA) is among the few Food and Drug Administration (FDA) approved synthetic materials for certain human clinical applications (e.g., degradable sutures, stents, wound dressings), which has been widely used as synthetic polymeric materials in scaffold fabrication. ${ }^{[29]}$ However, PLLA lacks functional groups for biomolecule conjugation.
Here, we synthesized a series of PLLA-based graft copolymers poly(L-lactic acid)-graft-poly(hydroxyethyl methacrylate) (PLLA-g-PHEMA) to introduce PHEMA blocks to PLLA for the conjugation of peptides or proteins. The schematic synthesis procedure is illustrated in Figure 1. Briefly, hydroxyethyl methacrylate (HEMA) was first used as the initiator for the ring-opening polymerization of L-lactide to synthesize macromonomer MACRO-PLLA. The macromonomer MACROPLLA was then copolymerized with HEMA (which served as monomers in this step) through free radical polymerization to synthesize PLLA-g-PHEMA. ${ }^{[30]}$ The hydroxyls in PHEMA block were then converted into acrylic groups through their reaction with methacrylic anhydride using DMAP/TEA chemistry, forming PLLA-g-PHEMA-acrylic. The chemical structures of these materials were confirmed by ${ }^{1} \mathrm{H}$-Nuclear Magnetic Resonance spectroscopy (Figure S1-3, Supporting Information). Advantageously, PLLA-g-PHEMA-acrylic is also biodegradable. Therefore, we have successfully synthesized biodegradable and functionalized PLLA-based copolymers for the fabrication of functional NF-HMS (FNF-HMS).

\subsection{Fabrication of FNF-HMS from PHEMA-g-PLLA-acrylic}

An important advantage of the newly synthesized functional block copolymers is their capability to self-assemble into advanced structures at multiple scales. When subjected to emulsification, phase separation, solvent extraction and freeze-drying processes, ${ }^{[27]}$ the block copolymers self-assembled into functional nanofibrous hollow microspheres (FNF-HMS) (Figure 2). Experimentally, the polymer was first dissolved in tetrahydrofuran (THF) at a concentration of $2 \% \mathrm{w} / \mathrm{v}$ at $50{ }^{\circ} \mathrm{C}$. Glycerol was then added gradually to emulsify the polymer solution into liquid microspheres via rigorous stirring at $50{ }^{\circ} \mathrm{C}$. Because a relative large amount of glycerol (more than three times the volume of the polymer solution) was gradually added into the rigorously stirred polymer solution, the initially dispersed phase of glycerol in the polymer solution transitioned into the continuous phase. This phase inversion led to the formation of "water-in-oil-inwater" (W/O/W) type double emulsion (glycerol-in-Polymer/ THF-in-glycerol) (Figure 2A). Although double emulsions are generally unstable, the block copolymers were believed to self-assemble to lower the associated free energy at the $\mathrm{W} / \mathrm{O} / \mathrm{W}$ interfaces and stabilize the double emulsions. Upon quenching in liquid nitrogen and the subsequent extraction of the solvent and glycerol, double emulsions became hollow microspheres.

The structure of the formed microspheres was characterized using scanning electron microscopy (Figure 2B-D). At the nano-scale, the copolymers were thermally induced to phase separate into nanofibers (diameters ranging from 50 to $500 \mathrm{~nm}$ ), mimicking the ECM collagen fiber structure. At the micro-scale, FNF-HMS had a hollow structure with an opening on the shell (which is approximately $20 \mu \mathrm{m}$ in diameter). This hollow structure can not only lower the density of the scaffold for faster degradation, but also facilitate cell seeding inside the microspheres for more robust tissue formation. At the macroscale, the microsphere suspension is injectable, facilitating 
<smiles>C=C(C)C(=O)OCCO</smiles>

HEMA<smiles>CC1OC(=O)C(C)OC1=O</smiles>

L-lactide<smiles>C=C(C)C(=O)OCCOC(C)=O</smiles><smiles>C=C(C)C(=O)OCCO</smiles>

AIBN, 1,4-dioxane $60{ }^{\circ} \mathrm{C}$

MACRO-PLLA<smiles>CC(O)C(=O)OC(C)C(=O)CCOC(=O)C(C)(C)CC(C)(C(=O)OCCO)C(=O)OCCO</smiles><smiles>C=C(C)C(=O)OC(=O)C(=C)SC(C)C(C)C(C)C</smiles>

1,4-dioxane

PHEMA-g-PLLA<smiles>C=C(C)C(=O)OCCOC(=O)C(C)(C)CC(C)(C)C(=O)OCCC(=O)C(C)OC(=O)C(C)OC(=O)C(=C)C</smiles>

PHEMA-g-PLLA-acrylic

Figure 1. Synthesis route of functional PLLA-based block copolymer PHEMA-g-PLLA-acrylic.

the regeneration of irregularly shaped tissue defects through minimally invasive procedures. These data demonstrated for the first time that these branched copolymers (instead of starshaped polymers) are able to form FNF-HMS with desirable structures as cell carriers for tissue engineering.
2.3. Establish "Thiol-ene" Click Reaction Between Biomolecules and FNF-HMS

In addition to the self-assembly capabilities at multiple scales, the novel functional block copolymers can facilitate bioconjugation 

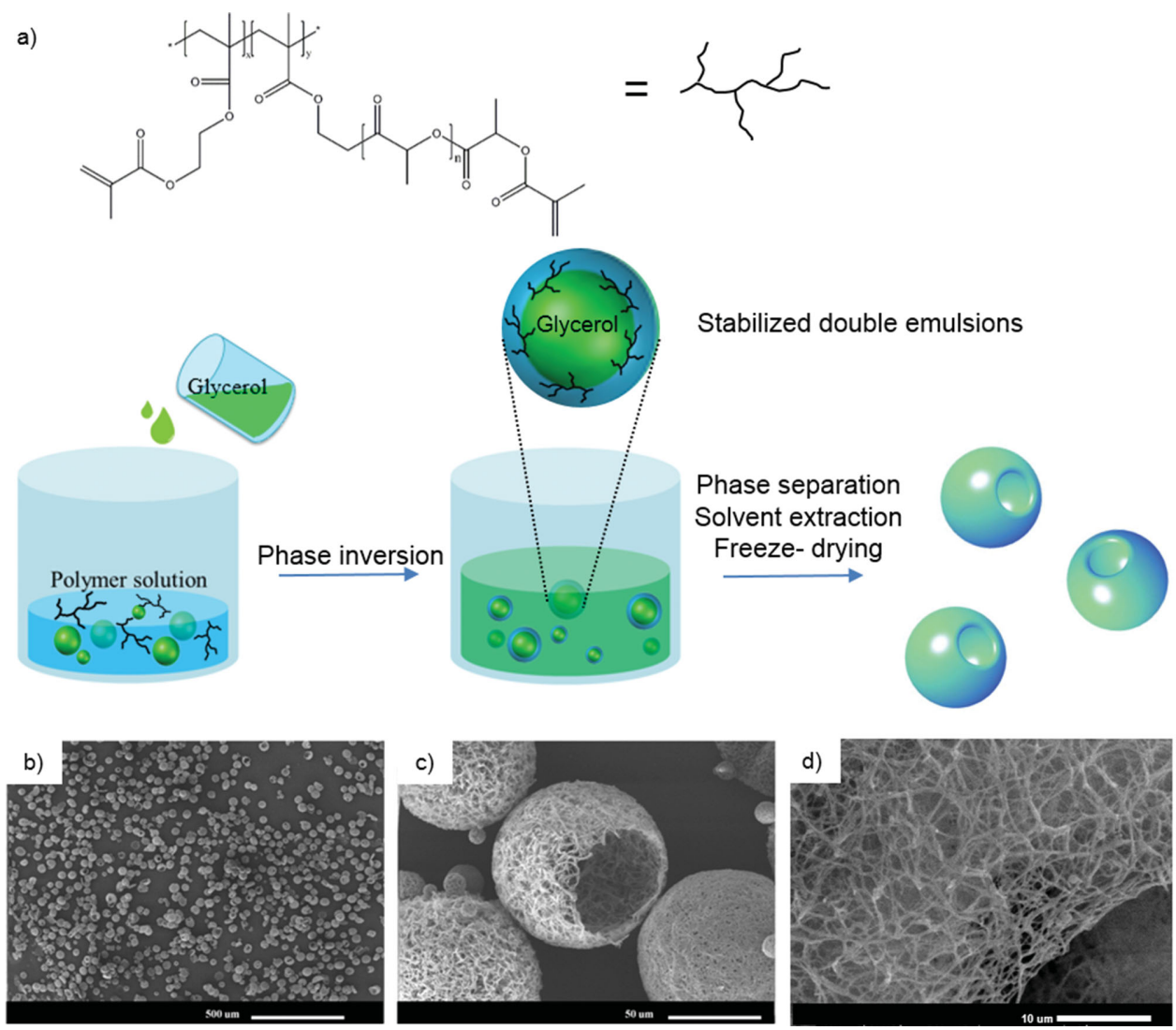

Figure 2. a) A schematic illustration showing the emulsification and phase separation techniques to fabricate FNF-HMS. b-d) SEM micrographs of FNF-HMS fabricated from a PHEMA-g-PLLA-acrylic copolymer.

with thiolated peptides through "thiol-ene" click reaction. To facilitate the click reaction, an additional cysteine (with one thiol side group) was added to the C-terminal of the selected peptides. The click reaction was performed through the nucleophile-mediated process due to its mild reaction conditions at room temperature. ${ }^{[31]}$ Tris(2-carboxyethyl)phosphine (TCEP) is employed as the nucleophile, which is also a reducing agent and can prevent the disulfide bonding between peptides. ${ }^{[32]}$ Due to the mild reaction conditions, the physical structure of FNF-HMS was unchanged after the click reaction (Figure S4, Supporting Information).

To visualize the conjugation sites and distribution of the conjugated molecules, fluorescent moieties were conjugated with FNF-HMS for confocal imaging and observation (Figure 3). Experimentally, thiol-PEG-biotin was conjugated to FNF-HMS, which subsequently bound FITC-tagged avidin through biotinavidin interaction (Figure 3A). Therefore, fluorescent signals were emitted from the conjugation sites. To minimize staining background due to non-specific binding of FITC-tagged avidin to FNF-HMS, the biotin-conjugated FNF-HMS were incubated with $10 \%$ bovine serum albumin (BSA) solution prior to the biotin-avidin conjugation. According to confocal imaging (Figure 3B,C), the avidin-tagged FNF-HMS exhibited fluorescent signals throughout the shell of the microspheres, indicating that the conjugated moieties were distributed on the entire FNF-HMS. When FNF-HMS without biotin conjugation were subjected to the same procedures (in BSA solution and then mixed with FITC tagged avidin), no fluorescent signals were emitted from FNF-HMS, indicating that specific binding of FITC-avidin to FNF-HMS occurred.

Advantageously, the developed FNF-HMS can conjugate controllable amount of peptides to present different concentrations of ligands to cells (Figure 4A). This was achieved by adjusting the density of acrylic groups in the copolymers during the molecular synthesis. Specifically, a higher feeding ratio of HEMA to macromonomer (MACRO-PLLA) during the copolymerization step (Figure 1) will produce copolymers with a higher density of hydroxyls from the PHEMA blocks. Upon the conversion of hydroxyls into acrylic groups, the copolymer with a higher HEMA to MACRO-PLLA ratio will present a higher density of acrylic groups for conjugating more peptides (Figure 4A). For simplicity, we define AD (acrylic group density) to be the molar feeding ratio of HEMA to MACROPLLA during the copolymerization. Therefore, FNF-HMS fabricated from copolymers with a high AD can conjugate more peptides.

To demonstrate our control over conjugation densities, FNFHMS were fabricated from copolymers with different AD values $(\mathrm{AD}=$ HEMA/ MACRO-PLLA $=0.8,0.9$, and 0.95) for peptide conjugation. An excess amount of peptide CM10 was used for the conjugation with FNF-HMS to ensure a high conversion of acrylic groups. The peptide immobilized FNF-HMS was first qualitatively analyzed using Fourier Transform Infrared Spectroscopy (FTIR) (Figure 4B). According to FTIR, peaks at 1601.3 and $1641.5 \mathrm{~cm}^{-1}$ disappeared after the click reaction, 
a)
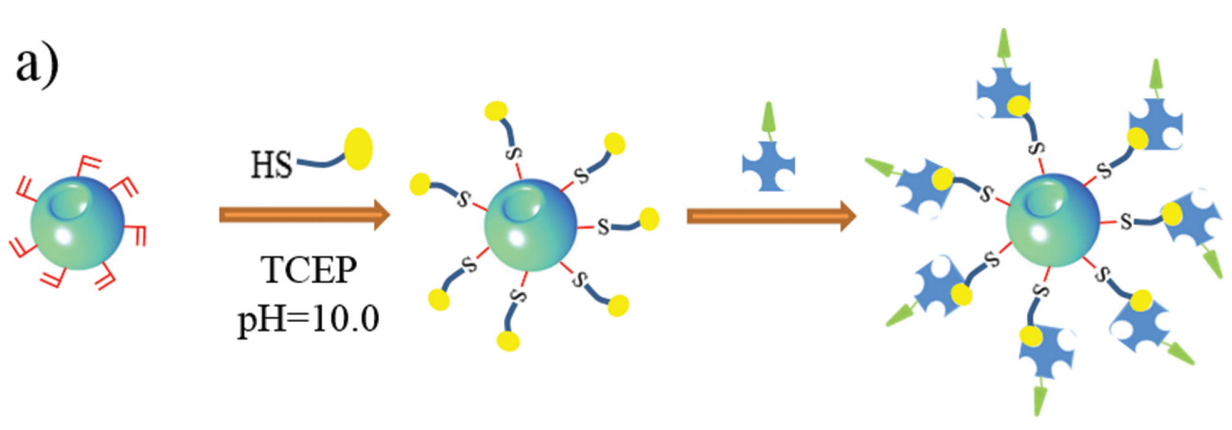

\section{TCEP}

$\mathrm{pH}=10.0$

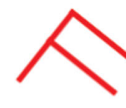

Acrylic groups

HS -

Thiol-PEG-biotin

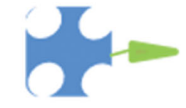

FITC-tagged avidin
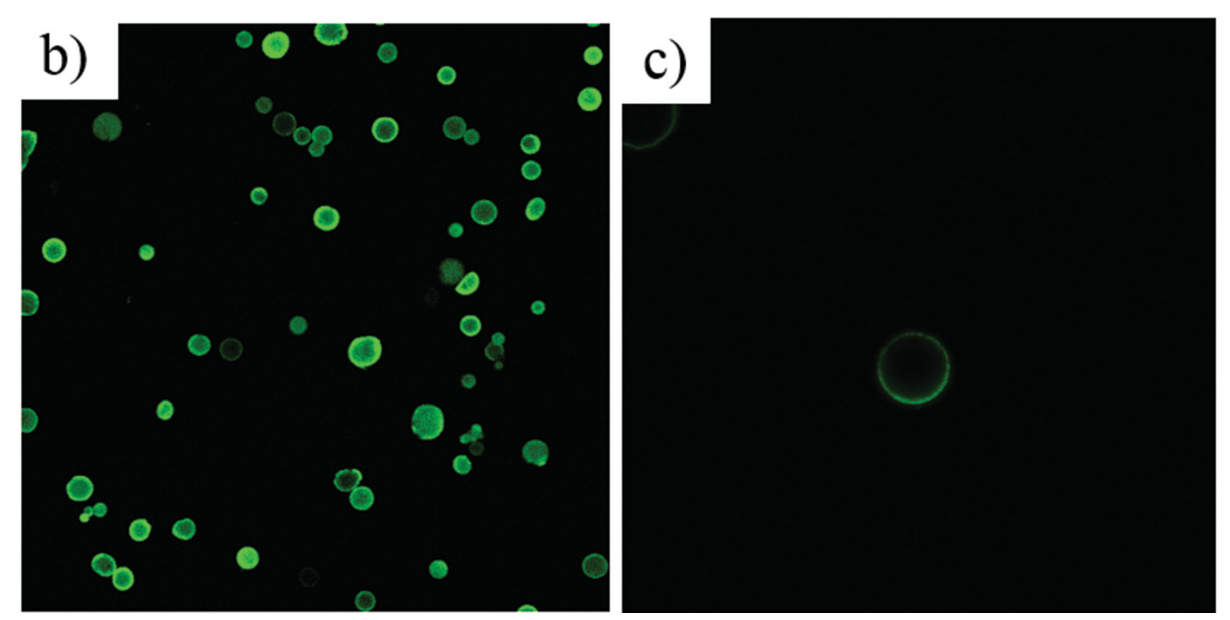

Figure 3. a) A schematic picture of the thiol-ene click reaction between FNF-HMS and thiol-PEG-biotin, and the subsequent binding with FITC-tagged avidin. b) A cross-sectional confocal image of FNF-HMS after fluorescent tagging of the conjugation sites. c) A cross-sectional confocal image of fluorescent-tagged FNF-HMS at a higher magnification, indicating that the click reaction occurred throughout the shells of FNF-HMS.

indicating that most of the acrylic groups on FNF-HMS were reacted. The conjugation was then evaluated quantitatively by amino acid analysis. As expected, FNF-HMS with a higher AD value conjugated more CM peptides (Figure 4C). FNFHMS with an AD of 0.8 can conjugate peptides at a density of $1.53 \mathrm{nmol} / \mu \mathrm{g}$, while FNF-HMS with an AD of 0.95 can conjugate peptides at a density of $11.16 \mathrm{nmol} / \mu \mathrm{g}$. Therefore, we can control the peptide density on FNF-HMS through the manipulation of copolymer composition.

\subsection{Conjugated FNF-HMS for Cartilage Regeneration}

Cytomodulin (CM) is a family of TGF- $\beta 1$ mimicking peptides. ${ }^{[18,19,33]}$ We selected a chondrogenic peptide CM10 (LIANAK) from the CM peptide family, conjugated it onto the developed FNF-HMS. To facilitate the conjugation of CM10 onto FNF-HMS, an additional cysteine was incorporated at the C-terminal of CM10 (therefore with an amino acid sequence of LIANAKC). In order to test the bioactivity of the conjugated CM10, FNF-HMS with or without CM10 were mixed with rabbit bone marrow-derived stem cells (BMSCs), and the in vitro chondrogenic differentiation of the stem cells and in vivo cartilage regeneration were examined. Rabbit BMSCs were selected because of their capability to differentiate along several lineages including chondrogenesis and osteogenesis.

In the in vitro study, FNF-HMS (AD =0.9) with or without CM10 conjugation were cultured with rabbit BMSCs in DMEM without TGF- $\beta 1$ for 3 weeks and the formed tissue constructs were examined using Safranin-O staining. Chondrogenic differentiation of BMSCs and typical cartilage formation were confirmed by positive Safranin-O staining for glycosaminoglycans (GAG) in the CM10-FNF-HMS group (Figure 5A,B), while no obvious cartilage-like tissue was found in the control FNF-HMS group without CM10 (Figure 5C,D). Therefore, we confirmed that CM10 peptide, after being conjugated onto FNF-HMS, remained biologically active and induced the chondrogenic differentiation of BMSCs in vitro.

In the in vivo study, FNF-HMS (AD =0.9) with or without CM10 conjugation were used as rabbit BMSC carriers for subcutaneous injection in mice. In the CM10-FNF-HMS group, cartilage formation was observed after injection for 2 weeks, demonstrated by strong safranin-O staining for GAG and immunohistochemical staining for collagen type II (Figure 6a,b). No obvious mineralization was noticed inside the tissue, revealed by negative von Kossa staining (Figure $6 \mathrm{c}$ ). In contrast, no cartilage was formed in the control FNF-HMS group without CM10, revealed by the negative Safranin-O staining for GAG and neg- 
a)

Acrylic group $\bigcirc \circ \bigcirc \bigcirc \bigcirc$ PLLA block $\bigcirc \circ \bigcirc \circ \circ \bigcirc$ PHEMA block

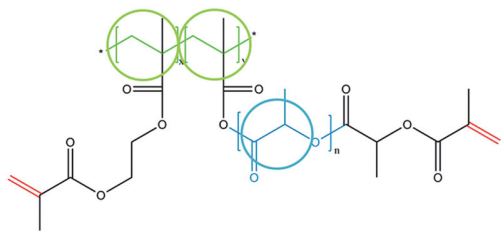

Higher HEMA percentage

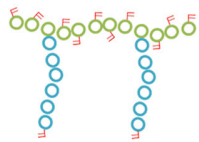

Self assembly

Phase separation Emulsification

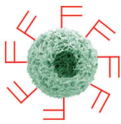

Higher acrylic group density
Lower HEMA percentage

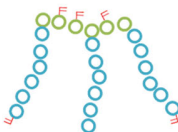

c)
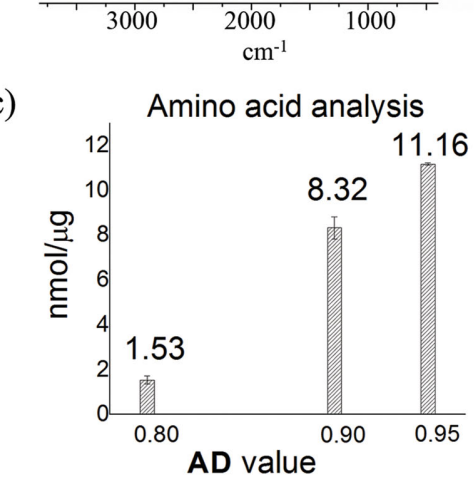

Figure 4. a) A schematic illustration showing the control over the density of functional acrylic groups (AD value) presented on the surface of FNF-HMS via the manipulation of copolymer compositions (HEMA block percentage). A higher HEMA block percentage (AD value) leads to a higher density of functional groups presented on the surface of FNF-HMS, and thus a higher density of peptides that can be conjugated. b) FITC spectrum of FNF-HMS before and after conjugation with CM10 peptides, showing the disappearance of peaks corresponding to acrylic groups (circled). C) Amino acid analysis of CM10-FNF-HMS at different conjugation densities.

ative immunohistochemical staining for collagen II (Figure 6d,e). Similarly, no obvious mineralization was observed according to the negative von Kossa staining (Figure 6f). Therefore, histological analysis following 2 weeks of subcutaneous injection revealed that TGF- $\beta 1$ mimicking peptide CM10 conjugated onto FNF-HMS remained bioactive and induced chondrogenic differentiation of rabbit BMSCs and ectopic cartilage formation.

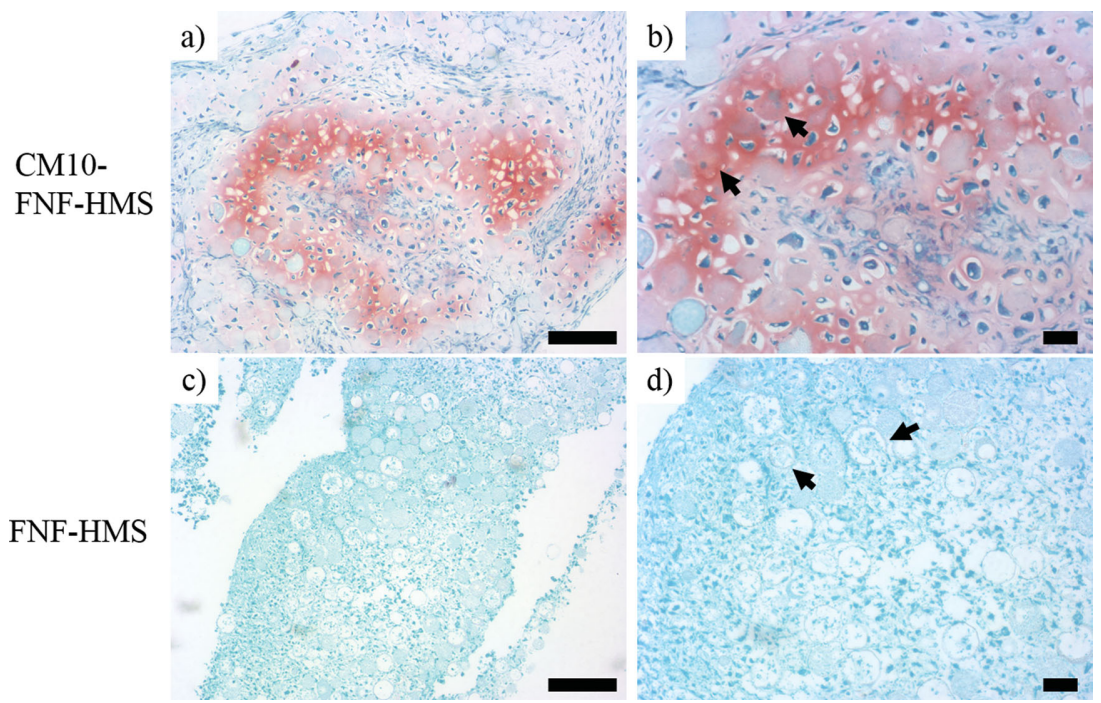

Figure 5. Chondrogenic differentiation of BMSCs were demonstrated by positive SO staining for GAC in CM10-FNF-HMS/BMSCs group $(a, b)$. The differentiated cells appeared round and encased in chondrocytic lacunae. Comparatively, in control group (FNF-HMS) (c,d), the staining was weak and no obvious cartilage-like tissue was found. In the high magnification images (b,d), arrows are used to indicate FNF-HMS with cells inside. Scale bar: $100 \mu \mathrm{m}$.

\subsection{P24 Conjugated FNF-HMS for Bone Regeneration}

The BMP-2 mimicking peptide P24 is known to promote osteogenesis. ${ }^{[17,34]}$ In this study, BMP-2 mimicking peptide P24 was also conjugated onto the newly developed FNF-HMS to evaluate the capacity of the P24-decorated FNF-HMS in inducing stem cell osteogenesis and bone regeneration. Experimentally, FNFHMS $(\mathrm{AD}=0.9)$ with or without peptide P24 were mixed with rabbit BMSCs and injected subcutaneously into nude mice. The specimens were harvested 5 weeks after injection and were examined histologically for new bone formation. H\&E staining revealed that P24- FNF-HMS supported robust, uniform bone formation throughout the specimen (Figure 7a). In the control group (blank FNF-HMS), there was significantly less and non-uniform bone formation (Figure 7d). While there was a very thin layer of bone tissue formation on the outer surface of the control specimen, likely benefited from the access to the host vasculature in these regions, there was only sparse bone nodule formation in the interior of the harvested tissue construct. Consistently, immunohistochemical staining showed a higher level of osteocalcin (a late marker of osteogenesis) ${ }^{[35]}$ in the P24-FNF-HMS group (Figure 7b) than in the control FNF-HMS group (Figure 7e). Furthermore, there were more and uniform mineralized domains (black) 
Safranin-O

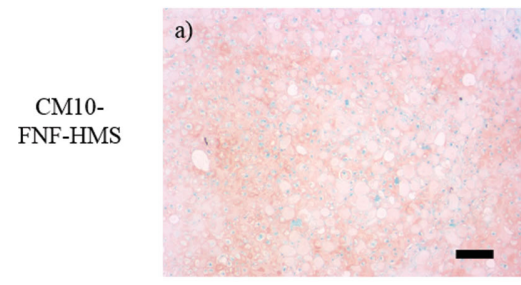

FNF-HMS

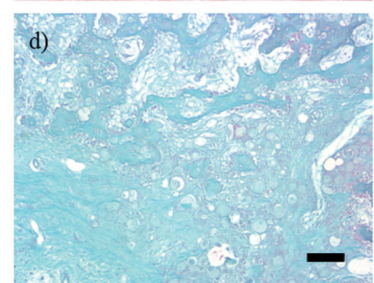

COL II

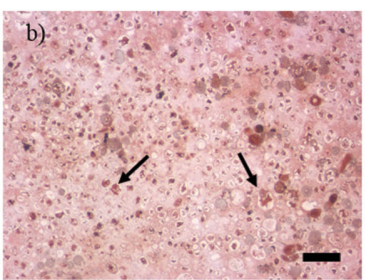

e)

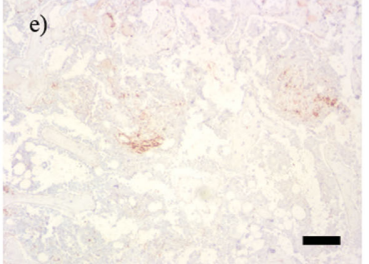

von Kossa

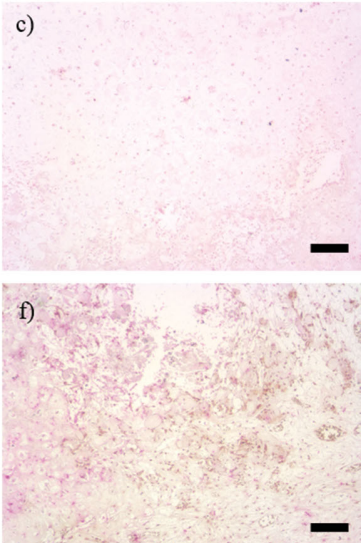

Figure 6. Hyaline cartilage formation after 2 weeks subcutaneous implantation was demonstrated by positive SO staining for GAC and immunohistochemical staining for collagen type II (COL II) in the CM10-FNF-HMS/BMSCs group (a\&b). No obvious mineralization was observed using von Kossa staining (c). In comparison, negative SO and immunohistochemical staining were seen in FNF-HMS/BMSCs group (d,e). There was no obvious mineralization in FNF-HMS/BMSCs group either (f). In image (b), arrows are used to indicate FNF-HMS with cells inside. Scale bar: $100 \mu \mathrm{m}$.

in the P24-FNF-HMS group (Figure 7c) than in the control FNF-HMS group (Figure 7f), revealed by von Kossa staining. All of the above results consistently indicate that the conjugated P24 on the FNF-HMS is biologically functional and shows similar osteogenic properties to BMP-2 in inducing osteogenic differentiation of rabbit BMSCs and the subsequent ectopic bone formation.

\section{Discussion}

Injectable microspheres can serve as cell carriers for tissue engineering. To deliver stem cells for regeneration, the microspheres should guide stem cells through the presentation of critical physical and chemical signals similar to those in the natural extracellular microenvironment. In natural ECM, collagen is the most abundant protein surrounding cells in the form of nanofibers, where GFs are bound. This complex structure may provide both physical cues and chemical stimuli to stem cells during development and wound healing. The delivery of potent growth factors is likely critical to the success of stem cell therapy, where nanofibers are likely an advantageous matrix structure for growth factor presentation. ${ }^{[10,23,36]}$ However, there has been no reported technology that can integrate synthetic NF structure and GF signals in injectable microspheres.

Recently, a novel technique has been developed to combine thermally induced phase separation with emulsification to generate nanofibrous hollow microspheres (NF-HMS). ${ }^{[27]}$ The nanofibers (with an average diameter of about $160 \mathrm{~nm}$ ) in the fabricated microspheres mimic the structural feature of collagen fibers at the nano-scale, and have a much higher porosity and surface area than those of traditional smooth surface microspheres. ${ }^{[27]}$ While linear PLLA can only form nanofibrous microspheres without a hollow core using this technique, starshaped PLLA can self-assemble into nanofibrous microspheres with a hollow core (NF-HMS) and openings on the shell of the
$\mathrm{H} \& \mathrm{E}$
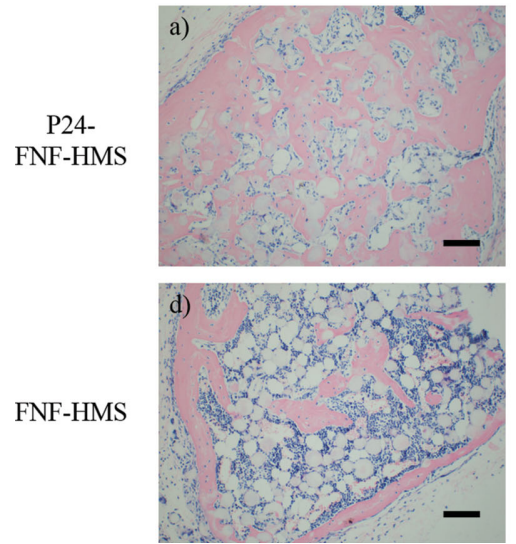

Osteocalcin
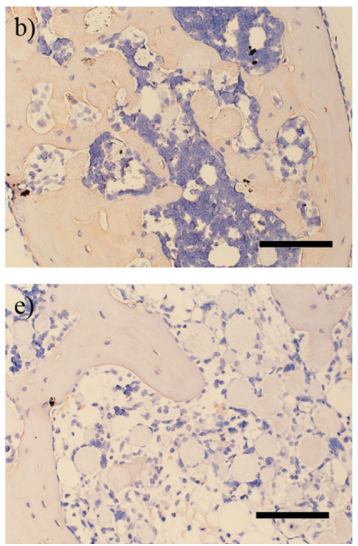
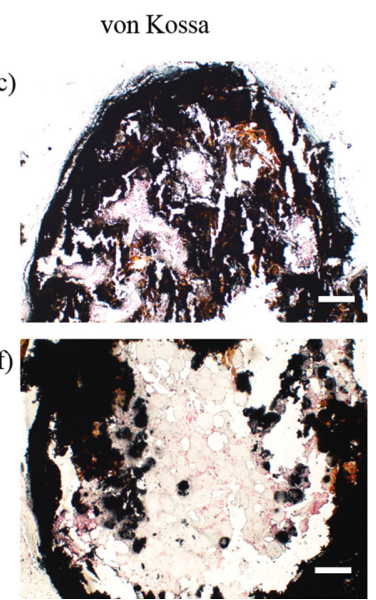

Figure 7. Histological analysis after $5 \mathrm{~W}$ mouse subcutaneous injection of BMP2-mimic P24 conjugated FNF-HMS (P24- FNF-HMS) or control FNF-HMS with rabbit BMSCs. P24- FNF-HMS reveal significantly more mature bone formation after $5 \mathrm{~W}$, shown by A) H\&E staining, B) osteocalcin immunohistochemical staining, and C) von Kossa staining for mineralization, compared to FNF-HMS (D-F). Scale bar: $100 \mu \mathrm{m}$. 
spheres. Desirably, the hollow structure of NF-HMS leads to an even higher porosity and provides more space for cell growth, ECM deposition, nutrient/waste transfer, and minimization of degradation products. ${ }^{[27]}$ The star-shaped molecular structure and the hydroxyl end groups on the SS-PLLA are considered important features for the assembly of NF-HMS. However, such an SS-PLLA lacks functional groups for biomolecule conjugation, limiting the presentation of biological signals on the NF-HMS assembled from such an SS-PLLA and therefore limiting their capacity of directing stem cell differentiation and tissue regeneration.

In this study, we have synthesized a series of novel PLLAbased functional graft copolymers PHEMA-g-PLLA-acrylic for the fabrication of functional NF-HMS (FNF-HMS) that allows GF immobilization. These graft copolymers, with the introduction of HEMA block onto PLLA, remain biodegradable and can be thermally induced to phase separate into nanofibers. ${ }^{[30]}$ Importantly, PHEMA-g-PLLA-acrylic can self-assemble into FNF-HMS during emulsification and phase separation procedures. We have demonstrated that PHEMA-g-PLLA-acrylic, similar to SS-PLLA, can stabilize double emulsions and form hollow microspheres, likely due to their hyper-branched molecular structure as well as hydroxyl groups from the HEMA blocks. Therefore, we have developed a new type of functional polymers to self-assemble into FNF-HMS, which enable us to introduce biological signals onto them for tissue regeneration.

FNF-HMS assembled from PHEMA-g-PLLA-acrylic have been shown capable of conjugating GF-mimicking peptides through "thiol-ene" click reaction for localized and sustained presentation of biological signals. Advantageously, the "thiol-ene" click reactions are orthogonal, tolerant to the presence of oxygen or water, and can proceed at an extremely high reaction rate with quantitative yields in highly polar solvents (such as water) in the absence of catalyst, ${ }^{[37]}$ which is desired for medical applications. While the conjugation can be performed prior to scaffold fabrication, we prefer to conjugate GF-mimicking peptides to the polymer following microsphere assembly. Through this strategy, the conjugation will occur only at the surface of the nanofibers where cell-matrix interactions take place. In addition, thiolated biomolecules can be conveniently mixed with FNF-HMS for conjugation prior to use, which allows for quick and easy examination of various biomolecules on FNF-HMS for tissue engineering or other applications. Furthermore, the conjugation densities can be easily controlled through the molecular synthesis of the functional copolymer (Figure 4). Copolymers with a higher AD value can conjugate a large amount of the peptide to present more concentrated signals to cells if desired. Therefore, targeted dosing of GF-mimicking peptides can be easily presented at the surface of the developed FNF-HMS.

GF mimics conjugated FNF-HMS, combining biochemical signals from GF mimics and nano-topographical features of nanofibers, are demonstrated to be highly advantageous in modulating stem cells for tissue regeneration in this work. The novel peptide-decorated FNF-HMS have advantageous properties for tissue regeneration in several aspects. First, the NF structure facilitates cell attachment, ${ }^{[22]}$ which likely facilitates the interactions between the immobilized GF mimics and the receptors on the stem cells. Second, the density of the localized GF signals (i.e., the dosing of GF mimics) can be utilized to manipulate the cell-ligand interactions. Third, GF signaling molecules, immobilized on FNF-HMS, can provide sustained interactions with the attached cells due to their slower degradation or internalization by cells. ${ }^{[8]}$ Fourth, unlike soluble GFs that might diffuse to the unintended surrounding tissues, ${ }^{[12]}$ tethered GFs only affect the adhered cells/tissues, minimizing undesired ectopic complications. Lastly, the high porosity and injectability of FNF-HMS allow for fast microsphere degradation and tissue repair in irregularly shaped defects through a minimally invasive surgical procedure.

While the injectable FNF-HMS are novel and capable of localized delivery of GF-mimicking peptides, an important question was whether the GF-mimicking peptides were bioactive after conjugation and whether the peptide-decorated FNFHMS could induce the aimed tissue regeneration. In this study, two different GF-mimicking peptides were conjugated onto the FNF-HMS and their potentials to induce the respective cartilage and bone regeneration were evaluated. While it was reported that soluble TGF- $\beta 1$ mimicking CM peptides were not able to induce chondrogenic differentiation of stem cells, ${ }^{[21]}$ we showed that CM conjugated FNF-HMS effectively induced BMSC chondrogenesis in both in vitro and in vivo studies. This could be attributed to the following advantages of using FNFHMS for cartilage regeneration. First, the nanofibers of FNFHMS could induce the chondrocyte phenotype and promote the early chondrogenic commitment of BMSCs. This was supported by our previous observation that cells showed a rounded shape on nanofibers, which is indicative of the chondrocyte phenotype and the commitment of the stem cells to the chondrogenic lineage. ${ }^{[28]}$ Second, the 3D culture of BMSCs on FNFHMS might support a long-term cartilaginous development. Different from a 2D cell culture, FNF-HMS allowed BMSCs to attach three-dimensionally, which further facilitated the chondrocyte phenotype and chondrogenesis (Figure S5, Supporting Information). These results support that FNF-HMS provide synergistic effect with TGF- $\beta 1$ signals to effectively promote BMSCs chondrogenesis. In addition to TGF- $\beta 1$ mimicking peptides, the BMP-2 mimicking peptide (P24) was conjugated onto the FNF-HMS and was shown to enhance BMSC osteogenesis and bone formation. Therefore, the FNF-HMS are likely an advanced delivery vehicle for a variety of GF-mimicking peptides. In the future, FNF-HMS will be utilized to deliver more types of peptides, biomolecules and their various combinations for diagnostic and therapeutic applications. For instance, FNFHMS conjugated with different peptides/biomolecules can be mixed, or multiple peptides/biomolecules can be conjugated onto the same FNF-HMS to achieve co-delivery. Such strategies aim to coordinate multiple biological signals to synergize their biological effects for the intended applications.

\section{Conclusion}

In this study, we have successfully developed novel FNF-HMS, which can conjugate GF-mimicking peptides with retained bioactivity, recapitulating both the geometrical and biochemical aspects of natural cell microenvironment to modulate stem cell fate. Desirably, these FNF-HMS allow the delivery of stem 
cells through injection to repair tissue using minimally invasive procedures. Two different GF-mimicking peptides were conjugated onto the newly developed FNF-HMS, and both conjugated peptides were shown biologically active in directing the stem cells along their respective differentiation pathways. When the TGF- $\beta 1$ mimicking peptide was conjugated onto the FNF-HMS, the injectable cell carrier induced chondrogenic differentiation of BMSCs and cartilage formation. When the BMP-2 mimicking peptide was conjugated onto the FNF-HMS, the injectable cell carrier induced osteogenic differentiation of BMSCs and bone formation. The NF structure appeared to enhance the efficacy of the GF-mimicking peptides in stem cell fate regulation. The new FNF-HMS therefore can serve as an advanced and versatile injectable carrier platform. In the future, FNF-HMS may be utilized to present or deliver a variety of peptides/biomolecules in various combinations for diagnostic and therapeutic applications.

\section{Experimental Section}

Materials: Tetrahydrofuran (THF) and glycerol were purchased from Aldrich Chemical (Milwaukee, WI). (3s)-cis-3,6-dimethyl-1, 4-dioxane-2,5-dione (L-lactide) was purchased from Sigma-Aldrich Inc. (St. Louis, MO) and was purified by recrystallization from toluene. Stannous 2-ethylhexanoate $\left(\mathrm{Sn}(\mathrm{Oct})_{2}\right)$ and 2-hydroxyethyl methacrylate (HEMA) were purchased from Sigma-Aldrich Inc. (St. Louis, MO), and were distilled under reduced pressure prior to use. 1,4-Dioxane was purchased from Aldrich Chemical (Milwaukee, WI) and was dehydrated over molecular sieve. 2,2'-Azoisobutyronitrile (AIBN) was obtained from Sigma-Aldrich Inc. (St. Louis, MO) and was recrystallized from ethanol. Tris (2-carboxyethyl) phosphine hydrochloride (TCEP), 4-(Dimethylamino) pyridine (DMAP), triethylamine (TEA) and methacrylic anhydride were purchased from Sigma-Aldrich Inc. (St. Louis, MO) and used as received. Albumin from bovine serum was also purchased from Sigma-Aldrich (St. Louis, MO) and used as received. Deionized water was obtained with a Milli-Q water filter system from Millipore Corporation (Bedford, MA). Cytomodulin and P24 peptides were synthesized by GenicBio Limited, Shanghai, China. Thiol-PEG-biotin was purchased from NANOCS Inc. (New York, NY). FITC-tagged avidin was purchased from BS Biosciences (San Jose, CA). Alpha-Minimum Essential Medium was purchased from Invitrogen. Fetal bovine serum (FBS), penicillin-streptomycin, Dulbecco's phosphate-buffered saline (PBS), and trypsin-EDTA were purchased from Gibco BRL Products, Life Technologies (Grand Island, NY, USA).

Preparation of PHEMA-g-PLLA-acrplic: A series of PHEMA-g-PLLA copolymers were synthesized following our previous published methods. ${ }^{[30]}$ Briefly, PLLA-based macromonomers were synthesized using the ring opening polymerization of L-lactide (LLA) with hydroxyalkyl methacrylates (HEMA) as the initiator, and subsequently copolymerized with HEMA. The synthesized PLLA-based macromonomers are abbreviated as HEMA-PLLAX, where $X$ is the feeding molar percentage of HEMA over LLA (e.g., $X=5$ means the feeding ratio of HEMA/LLA $=5 \%$ ). The final graft copolymers are abbreviated as PHEMAY-g-PLLAX, where $Y$ indicates the feeding molar percentage of macromonomer (HEMA-PLLAX) over HEMA (e.g., Y = 10 means the feeding molar ratio of HEMA-PLLAX/HEMA $=10 \%$ ). The $X$ was used to control the graft chain length, and the $Y$ was used to control the average backbone sectional length between the adjacent two graft chains. These two parameters were together used to manipulate the spatial distribution of the acrylic groups in the functionalized graft copolymers. A typical functionalization reaction of the copolymer PHEMA10-g-PLLA10 (towards PHEMA10g-PLLA10-acrylic) is as follows: the copolymer $(5 \mathrm{mmol} \mathrm{OH}, 2.500 \mathrm{~g})$, methacrylic anhydride (10 mmol, $1.542 \mathrm{~g})$, DMAP (10 mmol, $1.222 \mathrm{~g})$ and TEA (10 mmol, $1.012 \mathrm{~g}$ ) were dissolved in 1,4-dioxane $(200 \mathrm{~mL})$ and left overnight stirring at room temperature. The filtered solution was precipitated by adding ethyl ether, and the polymer precipitate was dried under vacuum for 3 days at room temperature.

Preparation of FNF-HMS: PHEMA-g-PLLA-acrylic was dissolved in $20 \mathrm{ml}$ of $\mathrm{THF}$ at $50{ }^{\circ} \mathrm{C}$ with a concentration of $2.0 \%$ (wt/v). Under rigorous mechanical stirring (speed 7, MAXIMA, Fisher Scientific Inc.), glycerol $\left(50^{\circ} \mathrm{C}\right)$ was gradually added into the polymer solution, and the stirring continued for another $5 \mathrm{~min}$. The mixture was then quickly poured into liquid nitrogen. After $10 \mathrm{~min}$, ice/water mixture was added for solvent exchange for $24 \mathrm{~h}$. The spheres were then sieved and washed with distilled water several times to remove glycerol residue. The spheres were then lyophilized for $2 \mathrm{~d}$.

Conjugation with Peptides: The selected peptide (CM or P24) (10 equiv) and TCEP (1 equiv) were dissolved in deionized water and added to a vial containing the FNF-HMS (1 equiv acrylic unit). The contents of the vial were purged with $\mathrm{N}_{2}$ for 10 min and stirred for $2 \mathrm{~h}$ at room temperature. The FNF-HMS was then washed extensively using deionized water and lyophilized for $2 \mathrm{~d}$.

NMR Observation: ${ }^{1} \mathrm{H}$ spectra of the macromonomers and copolymers were recorded with an Inova 400 NMR instrument operating at $400 \mathrm{MHz}$ at room temperature using deuterated chloroform $\left(\mathrm{CDCl}_{3}\right)$ as the solvent.

GPC Measurements: The molecular weights of the macromonomers and copolymers were measured using a Waters gel permeation chromatograph (GPC) model 440. Tetrahydrofuran (THF) was used as the mobile phase at a flow rate of $1.0 \mathrm{~mL} / \mathrm{min}$. Molecular weight and polydispersity of the copolymers were calibrated with polystyrene standards.

ATR-FTIR Observation: The ATR-FTIR spectrums of FNF-HMS before and after click reaction were obtained with a Perkin Elmer 1800 FTIR spectroscopy, in the region from 400 to $4000 \mathrm{~cm}^{-1}$. During the measurement, the sample chamber was purged with nitrogen gas to reduce moisture and carbon dioxide content.

Scanning Electron Microscopy Observation: The surface morphology of the microspheres was examined using SEM (Philips XL30 FEG) with an accelerating voltage of $10 \mathrm{kV}$. The samples were coated with gold for $120 \mathrm{~s}$ using a sputter coater (DeskII, Denton vacuum Inc).

Confocal Imaging: To visualize the distribution of the conjugated peptides, thiol-PEG-biotin was conjugated to the FNF-HMS under the same click reaction conditions as for the conjugation of CM and P24 peptides. After extensive washing using deionized water, the FNF-HMSbiotin were treated with $10 \% \mathrm{w} / \mathrm{V}$ BSA solution first, and then with $10 \%$ W/v FITC-tagged avidin solution to generate FNF-HMS-biotin-avidin. The treatment with $10 \% \mathrm{w} / \mathrm{v}$ BSA solution was to prevent unspecific attachment of FITC-tagged avidin. The FNF-HMS-biotin-avidin were examined using confocal laser scanning microscopy (CLSM) (Nikon Eclipse $\mathrm{C1}$ ). FNF-HMS were also treated with BSA and FITC-tagged avidin solutions for confocal imaging as the control group. All the parameters including the laser intensity and gain were adjusted until fluorescent signals cannot be seen from the control group (FNF-HMS); then without changing the settings, FNF-HMS-biotin-avidin were observed.

Amino Acid Analysis: The sample was first hydrolyzed in $6 \mathrm{~N} \mathrm{HCl}$ at $110{ }^{\circ} \mathrm{C}$ for $24 \mathrm{~h}$. Then it was reacted with FDNB (1-fluoro-2, 4-dinitrobenzene) and was analyzed by high performance liquid chromatography (HPLC). The amount of conjugated peptides was calculated.

Cell Harvest and In Vitro Pellet Culture: Bone marrow-derived mesenchymal stem cells (BMSCs) of two-week-old New Zealand White rabbits were collected via aspiration from the femoral bone marrow using an 18-gauge syringe needle, collecting $10 \mathrm{~mL}$ of marrow into $1000 \mathrm{U}$ of heparin-containing maintenance media (high-glucose alpha-MEM (Gibco) containing 10\% fetal bovine serum (Gibco) and antibiotics (penicillin G, $100 \mathrm{U} / \mathrm{mL}$; streptomycin, $0.1 \mathrm{mg} / \mathrm{mL}$ )). The marrow was filtered through a cell strainer to exclude fatty tissues and blood clots, washed with PBS once and fresh media twice, and centrifuged at $2000 \mathrm{rpm}$ for $5 \mathrm{~min}$ after each wash. Rabbit BMSCs were collected and cultured in $75-\mathrm{cm}^{2}$ flasks in maintenance media at $37{ }^{\circ} \mathrm{C}$ under $5 \% \mathrm{CO}_{2}$. For the preparation of each pellet, $2 \times 10^{6}$ passage- 3 
rabbit BMSCs were trypsinized, mixed with $2 \times 10^{5}$ microspheres with a diameter of $20-60 \mu \mathrm{m}$, and cultured to evaluate chondrogenic differentiation

In Vitro Studies of CM10-conjugated FNF-HMS: the following two groups were studied: I) BMSCS+FNF-HMS conjugated with CM10 in incomplete chondrogenic medium without TGF- $\beta 1$ (DMEM containing $1 \times 10^{-7} \mathrm{~mol} / \mathrm{L}$ dexamethasone, ITS-A $(1000 \mathrm{mg} / \mathrm{L}$ insulin, $550 \mathrm{mg} / \mathrm{L}$ transferrin, $0.67 \mathrm{mg} / \mathrm{L}$ sodium selenite, $11000 \mathrm{mg} / \mathrm{L}$ sodium pyruvate), and $50 \mu \mathrm{g} / \mathrm{mL}$ ascorbate-2-phosphate). II) BMSCs+FNF-HMS in incomplete chondrogenic medium without TGF- $\beta 1$ as the control. The cells were cultivated at $37{ }^{\circ} \mathrm{C}$ in a humidified atmosphere with $5 \% \mathrm{CO}_{2}$ for $21 \mathrm{~d}$, changing the medium every $3 \mathrm{~d}$.

Subcutaneous Injection of Rabbit BMSCs and Microspheres (FNF-HMS Conjugated $w /$ or $w / 0$ CM10 or P24): All animal procedures were carried out under the guidelines of the Institutional Animal Care and Use Committee of the University of Michigan. Nude mice (6-8 weeks old, NU/NU, Charles River Laboratories USA) were anaesthetized with $2.5 \%$ isoflurane in balanced oxygen. The microspheres/BMSCs suspension was injected into subcutaneous pockets on both sides lateral to the dorsal midline using a 25-gauge needle. Each mouse received four injections, with each $100 \mu \mathrm{L}$ injection containing $4 \times 10^{6}$ cells mixed with $4 \times 10^{5}$ microspheres for cartilage study or $1.5 \times 10^{6}$ cells and $1 \times 10^{5}$ microspheres for bone study. The injections were randomly arranged. The tissues were collected after two weeks or five weeks, and the fibrous capsules were removed. The samples were used for histological and immunohistochemical examinations.

Histological and Immunohistological Analyses: For histological observation, sections were deparaffinized, rehydrated, and stained with H-E, Safranin O/Fast Green, or von Kossa. For P24 bone study, bone tissue samples were decalcified with 10\% EDTA for two weeks prior to paraffin embedding for $\mathrm{H} \& \mathrm{E}$ and immunohistochemical (IHC) staining. For IHC staining for type II collagen and osteocalcin, following deparaffinization and rehydration, slides were pretreated with pepsin solution (Fisher Scientific, USA) for 15 min, incubated with the primary antibody (mouse type II collagen antibody or rabbit osteocalcin antibody, both from Santa Cruz Biotechnology, Santa Cruz, CA) at 1:100 dilutions for $1 \mathrm{~h}$ and prepared using a cell \& tissue staining kit (R\&D Systems Inc., Minneapolis, MN, USA). All sections were counterstained with hematoxylin.

\section{Supporting Information}

Supporting Information is available from the Wiley Online Library or from the author.

\section{Acknowledgements}

The authors would like to acknowledge the financial support from the National Science Foundation (DMR-1206575: PXM), National Institutes of Health (Research Grants DE015384 and DE017689: PXM), and DOD (W81XWH-12-2-0008: PXM). MJG was partially supported by an NIH Training Grant (5T32DE007057) fellowship and a GAANN fellowship. Rabbits for cell isolation were a generous gift from Dr. Y. Eugene Chen's laboratory at the University of Michigan.

Received: August 2, 2014 Revised: September 16, 2014 Published online: October 13, 2014

[1] A. I. Caplan, J. Cell. Physiol. 2007, 213, 341.

[2] G. Wei, P. X. Ma, Adv. Funct. Mater. 2008, 18, 3568.

[3] a) F. M. Chen, M. Zhang, Z. F. Wu, Biomaterials 2010, 31, 6279; b) F. Ramirez, D. B. Rifkin, Matrix Biol. 2003, 22, 101; c) A. J. Singer, R. A. F. Clark, N. Engl. J. Med. 1999, 341, 738.
[4] F. M. Chen, R. M. Shelton, Y. Jin, I. L. C. Chapple, Med. Res. Rev. 2009, 29, 472

[5] a) G. Wei, Q. Jin, W. V. Giannobile, P. X. Ma, Biomaterials 2007, 28, 2087; b) G. Wei, Q. Jin, W. V. Giannobile, P. X. Ma, J. Controlled Release 2006, 112, 103.

[6] a) B. K. Mann, R. H. Schmedlen, J. L. West, Biomaterials 2001, 22, 439; b) C. C. Lin, K. S. Anseth, Adv. Funct. Mater. 2009, 19, 2325; c) G. A. Hudalla, W. L. Murphy, Adv. Funct. Mater. 2011, 21,1754 .

[7] a) K. S. Masters, Macromol. Biosci. 2011, 11, 1149; b) T. A. McCaffrey, D. J. Falcone, B. Du, J. Cell. Physiol. 1992 152,430 ; c) R. Ruppert, E. Hoffmann, W. Sebald, Eur. J. Biochem. 1996, 237, 295

[8] P. R. Kuhl, L. G. Griffith-Cima, Nat. Med. 1996, 2, 1022.

[9] a) R. Langer, J. Folkman, Nature 1976, 263, 797; b) M. Hossain, R. Irwin, M. J. Baumann, L. R. McCabe, Biomaterials 2005 26, 2595.

[10] Y. Zhao, M. Tanaka, T. Kinoshita, M. Higuchi, T. Tan, J. Controlled Release 2010, 147, 392.

[11] X. Huang, C. S. Brazel, J. Controlled Release 2001, 73, 121.

[12] K. S. Cahill, J. H. Chi, A. Day, E. B. Claus, JAMA 2009, 302, 58.

[13] a) C. D. Hodneland, Y. S. Lee, D. H. Min, M. Mrksich, Proc. Natl. Acad. Sci. U.S.A. 2002, 99, 5048; b) K. Merrett, C. M. Griffith, Y. Deslandes, G. Pleizier, M. A. Dubé, H. Sheardown, J. Biomed. Mater. Res. Part A 2003, 67, 981.

[14] T. R. Chan, P. J. Stahl, S. M. Yu, Adv. Funct. Mater. 2011, 21, 4252.

[15] a) L. D. D. Andrea, A. D. Gatto, L. D. Rosa, A. Romanelli, C. Pedone, Curr. Pharma. Design 2009, 15, 2414; b) K. Tashiro, G. C. Sephel, B. Weeks, M. Sasaki, G. R. Martin, H. K. Kleinman, Y. Yamada, J. Biol. Chem. 1989, 264, 16174; c) E. S. Place, N. D. Evans, M. M. Stevens, Nat. Mater. 2009, 8, 457.

[16] Y. Suzuki, M. Tanihara, K. Suzuki, A. Saitou, W. Sufan, Y. Nishimura, J. Biomed. Mater. Res. 2000, 50, 405.

[17] K. Aoki, N. Alles, N. Soysa, K. Ohya, Adv. Drug Delivery Rev. 2012 64, 1220.

[18] a) S. Basu, M. Kumar, J. P. N. Chansuria, T. B. Singh, R. Bhatnagar, V. K. Shukla, Int. J. Surg. 2009, 7, 460; b) H. J. Lam, S. Li, N. Lou, J. Chu, R. S. Bhatnagar, "Synthetic peptides cytomodulin-1 (CM-1) and cytomodulin-2 (CM-2) promote collagen synthesis and wound healing in vitro", presented at, Engineering in Medicine and Biology Society, 2004. IEMBS '04. 26th Annual International Conference of the IEEE, 1-5 Sept. 2004.

[19] A. Mittal, R. Kumar, D. Parsad, N. Kumar, J. Tissue Eng. Regen. Med. 2012, $n / a$

[20] M. J. Kim, B. Lee, K. Yang, J. Park, S. Jeon, S. H. Um, D. I. Kim, S. G. Im, S. W. Cho, Biomaterials 2013, 34, 7236.

[21] J. N. Renner, J. C. Liu, Biotechnol. Prog. 2013, 29, 1550.

[22] K. M. Woo, V. J. Chen, P. X. Ma, J. Biomed. Mater. Res. Part A 2003, $67 A, 531$

[23] Z. Zhang, J. Hu, P. X. Ma, Adv. Drug Delivery Rev. 2012, 64, 1129.

[24] E. Genové, C. Shen, S. Zhang, C. E. Semino, Biomaterials 2005, 26 3341.

[25] H. S. Yoo, T. G. Kim, T. G. Park, Adv. Drug Delivery Rev. 2009, 61, 1033.

[26] a) V. J. Chen, P. X. Ma, Biomaterials 2004, 25, 2065; b) G. Wei, P. X. Ma, J. Biomed. Mater. Res.: Part A 2006, 78, 306.

[27] X. Liu, X. Jin, P. X. Ma, Nat. Mater. 2011, 10, 398.

[28] J. Hu, K. Feng, X. Liu, P. X. Ma, Biomaterials 2009, 30, 5061.

[29] a) R. Zhang, P. Ma, Processing of polymer scaffolds: Phase separation, Vol. 715, Academic Press, San Diego 2001; b) F. Yang, R. Murugan, S. Wang, S. Ramakrishna, Biomaterials 2005, 26, 2603.

[30] X. Liu, P. X. Ma, Biomaterials 2010, 31, 259.

[31] C. E. Hoyle, C. N. Bowman, Angew. Chem. Int. Ed. 2010, 49, 1540. 
[32] M. W. Jones, G. Mantovani, S. M. Ryan, X. Wang, D. J. Brayden, D. M. Haddleton, Chem. Commun. 2009, 5272.

[33] R. S. Bhatnagar, J. J. Qian, United States Patent 5780436, 1998.

[34] A. Saito, Y. Suzuki, S. I. Ogata, C. Ohtsuki, M. Tanihara, Biochim. Biophys. Acta 2003, 1651, 60.

[35] P. V. Hauschka, Haemostasis 1986, 16, 258.
[36] a) Y. Nagai, L. D. Unsworth, S. Koutsopoulos, S. Zhang, J. Controlled Release 2006, 115, 18; b) T. H. Kim, J. J. Kim, H. W. Kim, Biotechnol. Lett. 2014, 36, 383; c) C. M. Valmikinathan, S. Defroda, X. Yu, Biomacromolecules 2009, 10, 1084; d) Y. Z. Zhang, X. Wang, Y. Feng, J. Li, C. T. Lim, S. Ramakrishna, Biomacromolecules 2006, 7, 1049.

[37] A. B. Lowe, Polym. Chem. 2010, 1, 17. 